\title{
Coarsening Dynamics on $\mathbb{Z}^{d}$ with Frozen Vertices
}

\author{
M. Damron ${ }^{1}$, S. M. Eckner ${ }^{2}$, H. Kogan², C. M. Newman ${ }^{3}$, V. Sidoravicius ${ }^{4}$ \\ ${ }^{1}$ Department of Mathematics, Indiana University, Bloomington \\ ${ }^{2}$ Courant Institute of Mathematical Sciences \\ ${ }^{3}$ Courant Institute of Mathematical Sciences and NYU-Shanghai \\ ${ }^{4}$ IMPA, Rio de Janeiro, Brazil
}

October 11, 2018

\begin{abstract}
We study Markov processes in which \pm 1 -valued random variables $\sigma_{x}(t), x \in \mathbb{Z}^{d}$, update by taking the value of a majority of their nearest neighbors or else tossing a fair coin in case of a tie. In the presence of a random environment of frozen plus (resp., minus) vertices with density $\rho^{+}$(resp., $\rho^{-}$), we study the prevalence of vertices that are (eventually) fixed plus or fixed minus or flippers (changing forever). Our main results are that, for $\rho^{+}>0$ and $\rho^{-}=0$, all sites are fixed plus, while for $\rho^{+}>0$ and $\rho^{-}$very small (compared to $\rho^{+}$), the fixed minus and flippers together do not percolate. We also obtain some results for deterministic placement of frozen vertices.
\end{abstract}

\section{Introduction}

In this work we study the long time behavior of Markov processes, primarily in continuous time, whose states assign either +1 or -1 , called a spin value, to each vertex of the $d$-dimensional lattice $\mathbb{Z}^{d}$. We will discuss two types of processes, a much studied one denoted $\sigma(t)=\left(\sigma_{x}(t): x \in \mathbb{Z}^{d}\right)$ and then a modified one, denoted $\sigma^{\prime}(t)$, in which some vertices are "frozen" - that is, their spin values are not allowed to change.

Before giving complete definitions of $\sigma(t)$ and $\sigma^{\prime}(t)$, we give brief descriptions and motivations. $\sigma(t)$ has an energy-lowering dynamics with energy of the form $-\sum^{*} \sigma_{x} \sigma_{y}$, where $\sum^{*}$ denotes the sum over nearest neighbor pairs of vertices. Here, energy is lowered at the update of $\sigma_{x}$ if its value is changed to agree with a strict majority of neighbors. The modified process $\sigma^{\prime}(t)$ basically corresponds to $\sigma(t)$ in a random environment where randomly or deterministically selected vertices are frozen from time zero, some plus and some minus.

There are two distinct motivations for studying $\sigma^{\prime}$. One, explained in more detail below, comes from the usual $\sigma$ process, with random initial state, but on a slab, say $\mathbb{Z}^{2} \times\{0, \ldots, k-1\}$, rather than on $\mathbb{Z}^{d}$. Here, random rectangular regions of the form $R \times\{0, \ldots, k-1\}$ are fixed (thus effectively frozen) from time zero if they start with constant spin value. A second motivation comes from the energy-lowering dynamics of random-field models with energy $-\sum^{*} \sigma_{x} \sigma_{y}-\sum_{x} h_{x} \sigma_{x}$ 
where the $h_{x}$ 's are i.i.d. variables with common distribution $\rho$. Suppose, for example, that $\rho=$ $\rho^{+} \delta_{H}+\rho^{-} \delta_{-H}+\left(1-\rho^{+}-\rho^{-}\right) \delta_{0}$ with $H>2 d$, where $\delta_{r}$ denotes the unit point measure at $r$. The reader can then check that at vertex $x$ with $h_{x}=H$ (resp., $-H$ ), either from time zero or else after the first update at $x, \sigma_{x}(t)$ will be fixed plus (resp., minus) and thus effectively frozen. We proceed now to define our two processes, $\sigma$ and $\sigma^{\prime}$, and review some known results about $\sigma$.

\section{The two processes}

\subsection{The process $\sigma$}

The stochastic process $\sigma(t)=\sigma(t, \omega)$, where $\sigma_{x}(t)$ denotes the value of the spin at vertex $x \in \mathbb{Z}^{d}$ at time $t \geq 0$, starts from a random initial configuration $\sigma(0)=\left\{\sigma_{x}(0)\right\}_{x \in \mathbb{Z}^{d}}$, drawn from an independent Bernoulli product measure

$$
\mu_{\theta}\left(\sigma_{x}(0)=+1\right)=\theta=1-\mu_{\theta}\left(\sigma_{x}(0)=-1\right) .
$$

The system evolves in continuous time according to an agreement inducing dynamics: at rate 1, each vertex changes its value if it disagrees with more than half of its neighbors, and decides its spin value by tossing a fair coin in the event of a tie. This process corresponds to the zero-temperature limit of Glauber dynamics for a stochastic Ising model with ferromagnetic nearest-neighbor interactions and no external magnetic field (see, for example, [7] or [9]), having Hamiltonian (energy function)

$$
\mathcal{H}=-\sum_{\{x, y\}:\|x-y\|=1} \sigma_{x} \sigma_{y},
$$

where $\|x\|$ denotes the Euclidean norm of $x$.

More precisely, let $\mathcal{S}$ be the state space of configurations $\sigma$, i.e., $\mathcal{S}=\{-1,1\}^{\mathbb{Z}^{d}}$. The continuous time dynamics can be defined by means of independent, rate 1 Poisson processes (clocks), one assigned to each vertex $x$. If the clock at vertex $x$ rings at time $t$ and the change in energy

$$
\Delta \mathcal{H}_{x}(\sigma)=2 \sum_{y:\|x-y\|=1} \sigma_{x} \sigma_{y}
$$

is negative (respectively, positive), a spin flip (that is, a change of $\sigma_{x}$ ) is done with probability 1 (respectively 0). To resolve the case of ties when $\Delta \mathcal{H}_{x}(\sigma)=0$, each clock ring is associated to a fair coin toss and a spin flip is done with probability $1 / 2$, or equivalently $\sigma_{x}$ is made to be +1 (respectively, -1 ) if the coin toss comes up heads (resp., tails). Let $\mathbb{P}_{\text {dyn }}$ be the probability measure for the realization of the dynamics (clock rings and tie-breaking coin tosses), and denote by $\mathbb{P}_{\theta}=\mu_{\theta} \times \mathbb{P}_{\text {dyn }}$ the joint probability measure on the space $\Omega$ of initial configurations $\sigma(0)$ and realizations of the dynamics; an element of $\Omega$ is denoted $\omega$.

This process has been studied extensively in the physics and mathematics literature - primarily on graphs such as the hyperlattice $\mathbb{Z}^{d}$ and the homogeneous tree of degree $K, \mathbb{T}_{K}$. A physical motivation, which corresponds to the symmetric initial spin configuration, is the behavior of a magnetic system following a deep quench. A deep quench occurs when a system that has reached equilibrium at an initial high temperature $T_{1}$ is instantaneously subjected to a very low temperature $T_{2}$. Here we take $T_{1}=\infty$ and $T_{2}=0$. For references on this and related problems see, for example, [7] or [9]. The main focus in the study of this model is the formation and evolution of boundaries 
delimiting same spin cluster domains. These domains shrink or grow or split or coalesce as their boundaries evolve. This model is often referred to as a model of domain coarsening.

A fundamental question is whether the system has a limiting configuration, or equivalently does every vertex eventually stop flipping? Whether

$$
\lim _{t \rightarrow \infty} \sigma_{x}(t)
$$

exists for almost every initial configuration, realization of the dynamics and for all $x \in \mathbb{Z}^{d}$ depends on $\theta$ and on the dimension $d$. We refer to the existence of the limit (3) as fixation at $x$.

Nanda, Newman and Stein [7] investigated this question when $d=2$ and $\theta=\frac{1}{2}$ and found that the limit does not exist; that is, every vertex flips infinitely often. Their work extended an old result of Arratia [1, who showed the same happens on $\mathbb{Z}$ (for $\theta \neq 0$ or 1 ). It is an open problem to determine what happens for $d \geq 3$ and $\theta=1 / 2$. One important consequence of the methods of 7 is that if each vertex of the graph has odd degree (for example, on $\mathbb{T}_{K}$ for $K$ odd), then $\sigma_{x}(\infty)$ does exist for almost every initial configuration, realization of the dynamics and every vertex $x$.

Another question of interest is whether sufficient bias in the initial configuration leads the system to reach consensus in the limit. That is, does there exist $\theta_{*} \in(0,1)$, such that for $\theta \geq \theta_{*}$,

$$
\forall x \in G, \mathbb{P}_{\theta}\left(\exists T=T(\sigma(0), \omega, x)<\infty \text { so that } \sigma_{x}(t)=+1 \text { for } t \geq T\right)=1 ?
$$

We will refer to (4) as fixation to consensus. It was conjectured by Liggett [10] that fixation to consensus holds for all $\theta>\frac{1}{2}$. Fontes, Schonmann and Sidoravicius [5] proved fixation to consensus for all $d \geq 2$ and $\theta_{*}$ strictly less but very close to 1 . On $\mathbb{T}_{3}$, however, Howard [6] showed that for some $\theta>1 / 2$, the system does not fixate to +1 consensus.

\section{$2.2 \sigma$ on slabs}

In [3] and [4, Damron, Kogan, Newman and Sidoravicius studied coarsening started from a configuration sampled from $\mu_{\theta}$ on two-dimensional slabs of finite width $k$ with free boundary conditions, which we denote as $\mathrm{Slab}_{k}$. These are graphs with vertex set $\mathbb{Z}^{2} \times\{0,1, \ldots, k-1\}(k \geq 2)$ and edge set $\mathcal{E}_{k}=\left\{\{x, y\}:\|x-y\|_{1}=1\right\}$. Their work was motivated by the question of whether there are vertices that fixate for $d \geq 3$ (and for which values of $d$ ). It has been suggested by computational results of Spirin, Krapivsky and Redner [12] that some vertices do indeed fixate.

The work in [3] and [4] on slabs highlights the possible diferences in long time behavior between $\mathbb{Z}^{2}$ and $\mathbb{Z}^{3}$. The authors showed that if $k=2$ the system fixates with both free and periodic boundary conditions; if $k=3$ with periodic boundary conditions the system also fixates; for all $k \geq 4$ with periodic boundary conditions some vertices fixate for large times and some do not, and the same result holds for all $k \geq 3$ with free boundary conditions. We call vertices which change spin sign forever flippers. One question which remains open, is whether the set of flippers percolates (contains an infinite component).

On Slab 3 , each $v \in \mathbb{Z}^{2} \times\{0\}$ or $v \in \mathbb{Z}^{2} \times\{2\}$ has five neighbors, so by a variant of the arguments of Nanda, Newman, Stein [7, $v$ fixates almost surely. Therefore on this graph, flippers can only exist in $\mathbb{Z}^{2} \times\{1\}$. On the other hand, if the initial configuration on Slab 3 is chosen according to a symmetric Bernoulli product measure, then by the Ergodic Theorem there are at $t=0$ infinitely many pillar-like same-spin formations (say, $2 \times 2 \times 3$ blocks) that are stable under the dynamics. These pillars are analogous to frozen vertices on $\mathbb{Z}^{2}$ in our new process $\sigma^{\prime}$. A general version of this new process on $\mathbb{Z}^{d}$ is presented in the following section. 


\subsection{The process $\sigma^{\prime}$}

In this subsection we define a new stochastic process on $\mathbb{Z}^{d}$, which we denote by $\sigma^{\prime}(t)$, in which some vertices are frozen for all time and the others are not. There are two basic versions of $\sigma^{\prime}$, which we will call disordered and engineered, according to whether the frozen vertices are chosen randomly or deterministically. The initial configuration of the disordered $\sigma^{\prime}$ will be assigned as follows. Fix $\rho^{+}, \rho^{-} \geq 0$ with $\rho^{+}+\rho^{-} \leq 1$ and pick three types of vertices (frozen plus, frozen minus and unfrozen) by i.i.d. choices with respective probabilities $\rho^{+}, \rho^{-}$and $1-\left(\rho^{+}+\rho^{-}\right)$. Once the frozen vertices have been chosen and assigned a spin value, the non-frozen vertices will be assigned spin values arbitrarily. (In other words, the theorems we prove will be valid for all choices of such spin values.) We will denote by $\mathbb{P}=\mathbb{P}_{\rho^{+}, \rho^{-}} \times \mathbb{P}_{\text {dyn }}$ the overall probability measure where $\mathbb{P}_{\rho^{+}, \rho^{-}}$is the distribution for the assignment of frozen plus and minus vertices and $\mathbb{P}_{\mathrm{dyn}}$ is the distribution of the following dynamics for $\sigma^{\prime}(t)$. The continuous time dynamics is defined similarly to that of the $\sigma(t)$ process. Vertices are assigned independent, rate 1 Poisson clock processes and tie-breaking fair coins, and flip sign to agree with a majority of their neighbors. Frozen vertices, however, never flip regardless of the configuration of their neighbors. In the engineered $\sigma^{\prime}$ the frozen vertices are chosen deterministically while the non-frozen vertices are assigned values at time zero by i.i.d. choices with respective probabilities $\theta$ and $1-\theta$ for +1 and -1 .

As usual, we are interested in the long term behavior of this model depending on the dimension $d$, the densities of frozen vertices, $\rho^{+}, \rho^{-}$(or in the engineered version, the choice of frozen vertices), and the initial configuration of vertices, which we denote by $\sigma^{\prime}(0) . \sigma^{\prime}(0)$ may be regarded as an element of $\{-1,+1\}^{\mathbb{Z}^{d}}$ even though the frozen vertex spins are instantaneously replaced (at time $t=0+)$ by their frozen values. Note that the $\sigma^{\prime}$ processes for all possible choices of $\sigma^{\prime}(0)$ are coupled on a single probability space. When $\rho^{+}>0$ and $\rho^{-}>0$, almost surely there exist flippers. To see this, consider the following configuration for the case $d=2$, which has probability $\left(1-\rho^{+}-\rho^{-}\right)\left(\rho^{+}\right)^{2}\left(\rho^{-}\right)^{2}$ : the vertex labeled $v$ in Figure 1 below is not frozen, but has two frozen neighbors of spin +1 and two frozen neighbors of spin -1 , and thus flips infinitely often. Similar flippers, as well as more complicated clusters of flippers, occur for any $d$.

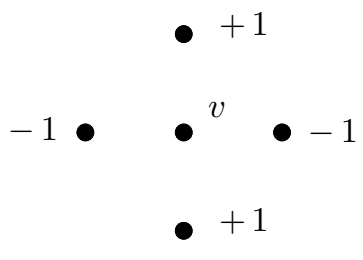

Figure 1: A vertex that flips infinitely often.

We conclude that $\sigma_{v}(t)$ need not have a limit as $t \rightarrow \infty$. So we are interested in whether the flippers percolate. Of course, one may study percolation of any of the three types of vertices (fixed plus, fixed minus or flippers) or of the union of two of the three. Theorem 3.2 below answers a question in this direction. 


\section{Main results}

Our first two theorems concern the disordered version of $\sigma^{\prime}$ and the last concerns engineered versions. The first theorem is a fixation to consensus result for the case of positive initial density of frozen +1 's and zero initial density of frozen -1 's. The second theorem is a more general result in which both $\rho^{+}, \rho^{-}>0$, but we require $\rho^{-}$to be much smaller than $\rho^{+}$. For this more general case we obtain that the set of flippers together with (eventually) fixed vertices of spin -1 does not percolate.

Theorem 3.1. Consider the disordered stochastic process $\sigma^{\prime}$ on $\mathbb{Z}^{d}$ for any $d$ and any $\rho^{+}>0$, with $\rho^{-}=0$. Then $\mathbb{P}\left(\right.$ the system fixates to +1 consensus for any $\left.\sigma^{\prime}(0)\right)=1$.

Theorem 3.2. Consider the disordered stochastic process $\sigma^{\prime}$ on $\mathbb{Z}^{d}$ for any $d$ and any $\rho^{+}>0$, with $\rho^{-}>0$ sufficiently small (depending on $\rho^{+}$and $d$ ). For an initial configuration $\sigma^{\prime}(0)$, denote by $\mathcal{C}\left(\sigma^{\prime}(0)\right)$ the collection of (eventually) fixed -1 vertices and flippers. Then $\mathcal{C}$ does not percolate:

$$
\mathbb{P}\left(\mathcal{C}\left(\sigma^{\prime}(0)\right) \text { contains an infinite component for some } \sigma^{\prime}(0)\right)=0 \text {. }
$$

Remark 3.1. Our main results, with essentially the same proofs, remain valid when the process $\sigma^{\prime}$ is modified in various ways. For example, the rules for breaking ties can be modified as long as there is strictly positive probability to update to +1 . Also the process can evolve in discrete time with synchronous updating. Another modification is to replace the two spin values, $\{-1,+1\}$, by $q$ values, say $\{1,2, \ldots, q\}$, as long as updates respect a majority agreement of neighbors on one value.

The next theorem concerns engineered versions of $\sigma^{\prime}$ in which the frozen vertices are all +1 and chosen deterministically while the other spin values at time zero are i.i.d. with probability $\theta$ to be +1 . Since in the disordered $\sigma^{\prime}$ there are infinitely many frozen vertices, it's natural to consider choosing infinitely many frozen +1 vertices forming a lower dimensional subset of $\mathbb{Z}^{d}$, such as $\mathbb{Z}^{d-k} \times \emptyset_{k}$ with $k, d-k \geq 1$, where $\emptyset_{k}$ denotes the origin in $\mathbb{Z}^{k}$.

Although we have no results to report for any of these situations, the next theorem concerns a slab approximation to the $k=1$ case where all the vertices in a codimension one hyperplane are frozen to +1 . Note that the frozen hyperplane separates $\mathbb{Z}^{d}$ into two graphs isomorphic to $\mathbb{Z}^{d-1} \times\{0,1,2, \ldots\}$ with $\mathbb{Z}^{d-1} \times\{0\}$ frozen to +1 , that evolve independently of each other.

Theorem 3.3. Consider the engineered stochastic process $\sigma^{\prime}$ on $\mathbb{Z}^{d-1} \times\{0,1,2, \ldots, K\}$, for $d \geq 2$ and $K \geq 1$, with all spins on $\mathbb{Z}^{d-1} \times\{0\}$ frozen to +1 and $\sigma^{\prime}(0)$ for other vertices chosen from $\mu_{\theta}$. Then for any $\theta>0$, with probability 1 the system fixates to +1 consensus.

\section{Proofs}

In this section we give the proofs of the theorems stated in Section 3 . Let $B_{L}=[-L, L]^{d}$ be the cubic box of side length $2 L+1$ centered at the origin and $B_{L}(x)=x+[-L, L]^{d}$ be the translated box centered at $x \in \mathbb{Z}^{d}$.

\subsection{Bootstrap percolation}

Following Fontes, Schonmann and Sidoravicius [5, Section 2], we describe the bootstrap percolation process that assigns configurations $\{u, s\}^{\mathbb{Z}^{d}}$ to a subset of $\mathbb{Z}^{d}$; here $u$ represents an unstable spin and $s$ represents a stable spin at a vertex. 
Definition 4.1. The d-dimensional $(u \rightarrow s)$ bootstrap percolation process with threshold $\gamma$, defined in a finite or infinite volume $\Lambda \subseteq \mathbb{Z}^{d}$, starting from the initial configuration $\eta(0) \in\{u, s\}^{\Lambda}$ is a cellular automaton which evolves in discrete time $t=0,1,2, \ldots$ such that at each time unit $t \geq 1$ the current configuration is updated according to the following rules. For each $x \in \Lambda$,

1. If $\eta_{x}(t-1)=s$, then $\eta_{x}(t)=s$.

2. If $\eta_{x}(t-1)=u$, and at time $t-1$ the vertex $x$ has at least $\gamma$ neighbors in $\Lambda$ in state $s$, then $\eta_{x}(t)=s$; otherwise, the spin at vertex $x$ remains unchanged; that $i s, \eta_{x}(t)=u$.

We will consider this process with threshold $\gamma=d$, as its evolution is close to our coarsening dynamics, and assume the initial configuration to be chosen from an independent Bernoulli product measure $P\left(\eta_{x}(0)=s\right)=p$, for $p$ small, on $\Lambda=\mathbb{Z}^{d}$. Note that by monotonicity of the dynamics, each $\eta_{x}(t)$ has a limit as $t \rightarrow \infty$.

Definition 4.2. A configuration $\eta \in\{u, s\}^{\Lambda}$ internally spans a region $B_{L}(x) \subset \Lambda$, if the bootstrap percolation restricted to $B_{L}(x)$, started from $\left.\eta\right|_{B_{L}}$, ends up with all vertices of $B_{L}$ in state s. We will denote by $\eta_{L}$ the subset $\left\{x \in B_{L}: \eta_{x}=s\right\}$ for such an $\eta$ and call it spanning.

The following proposition, an immediate consequence of results of Schonmann [11, provides a key ingredient for our proofs.

Proposition 4.1. [Schonmann] If $p>0$, then

$$
\lim _{L \rightarrow \infty} P\left(B_{L} \text { is internally spanned }\right)=1 .
$$

Remark 4.2. In [11] a variation of bootstrap percolation with threshold $\gamma=d$, called the modified basic model, is considered. Here, rule 2 is replaced by one which requires at least one neighbor in each of the $d$ coordinate directions from $x$ to have value $s$ in order that $x$ change from $u$ to s. For the modified basic model, the analogue of Proposition 4.1 remains valid - see [11, Proposition 3.2]. This will be used in the proof of Theorem 3.3.

\subsection{Preliminary lemmas}

We will make a comparison between the bootstrap percolation process on $\mathbb{Z}^{d}$ and our process $\sigma^{\prime}$ by mapping frozen plus spins to stable spins $s$, and all other spins to unstable spins $u$. In fact, we will compare only on finite regions which do not contain frozen minus vertices, so it is unimportant that these vertices are mapped to $u$. We say that a region $B_{L}(x)$ contains a spanning subset of frozen plus vertices if the configuration obtained by the above mapping internally spans $B_{L}(x)$.

Definition 4.3. $B_{L}(x)$ is entrapped if it contains a spanning subset of frozen plus vertices. It is captured if it is entrapped and all $2^{d}$ corners are frozen plus. It is $M$-captured $(M \in\{0,1, \ldots, L\})$ if it is entrapped and for each of the $2^{d}$ corners $C^{i},\left(i=1, \ldots, 2^{d}\right)$, and each coordinate direction $j=1, \ldots, d$ there is a frozen plus vertex within $B_{L}(x)$ of the form $C^{i, j}=C^{i}+m e^{j}$ with $|m| \leq M$ (where $e^{1}, \ldots, e^{d}$ are the standard basis vectors of $\mathbb{R}^{d}$ ) - see Figure 2. Note that captured is the same as 0-captured.

The notion of $M$-captured will be used (in Lemma 4.8) to guarantee that with high probability most of the vertices in the box $B_{L}$ (at least for $1 \ll M \ll L$ ) will fixate to +1 . Note that, although in the above definition $M=L$ is allowed, in Lemmas 4.7 and 4.8 we require $M<L$. Lemma 4.6 though, will allow us to choose $1 \ll M \ll L$ in the final proof, although we do not make use of this. Lemmas 4.3, 4.4 and 4.5. presented next, will be used in the proof of Theorem 3.1 . 


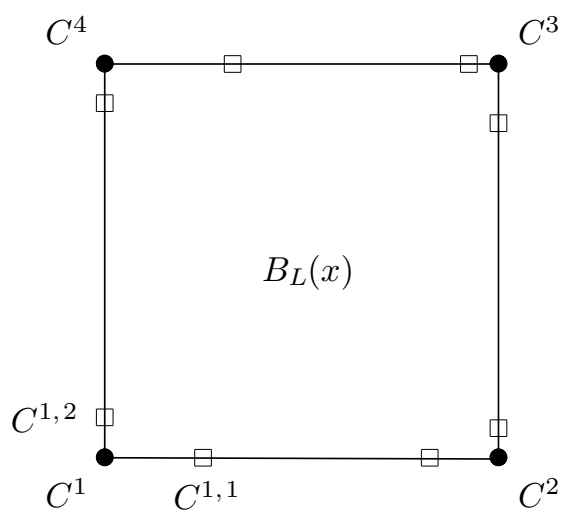

Figure 2: $B_{L}(x)$ is M-captured.

Lemma 4.3. Given $L$ and a spanning subset $\eta_{L}$ of $B_{L}$, consider the $\sigma^{\prime}$ process in $\mathbb{Z}^{d}$ with initial spins in $\eta_{L}$ taken as frozen plus and all others in $\mathbb{Z}^{d} \backslash \eta_{L}$ taken as minus but not frozen. Then

$$
\mathbb{P}\left(\text { for some } t \in[0,1],\left.\sigma^{\prime}(t)\right|_{B_{L}} \equiv+1\right)>0 .
$$

Proof. Since $\eta_{L}$ is a spanning subset of $B_{L}$, the corresponding bootstrap percolation process occupies all vertices of $B_{L}$ in a finite number of steps. Since the threshold $\gamma=d$, we can, with a small but positive probability, arrange the clock rings for $t \in(0,1)$ and tie-breaking coin tosses of the coarsening dynamics to mimic the dynamics of bootstrap percolation (in a much longer discrete time interval). Thus $\left.\sigma^{\prime}(t)\right|_{B_{L}} \equiv+1$ for some $t \in[0,1]$ with positive probability.

The next lemma strengthens the last one by showing that, if we allow the process to run until a large time, then with probability close to one all the vertices of $B_{L}$ will flip to +1 before that time. Let $\operatorname{Span}_{L}$ be the event that the frozen plus vertices span $B_{L}$ and there are no frozen minus vertices in $B_{L}$.

Lemma 4.4. Given $L<\infty$,

$$
\lim _{T \rightarrow \infty} \mathbb{P}\left(\exists t \in[0, T] \text { such that }\left.\sigma^{\prime}(t)\right|_{B_{L}} \equiv+1 \text { for all } \sigma^{\prime}(0) \mid \operatorname{Span}_{L}\right)=1 .
$$

Proof. Pick a maximal spanning subset $\eta_{L}$ (in some deterministic ordering of subsets) of frozen plus vertices of $B_{L}$. By Lemma 4.3 and attractiveness (monotonicity of the dynamics), there is an $\epsilon^{\prime}>0$ such that, for any $m$ and $\sigma^{\prime}(m)$ (consistent with the frozen vertices in $B_{L}$ ),

$$
\mathbb{P}\left(\text { for some } t \in[m, m+1],\left.\sigma^{\prime}(t)\right|_{B_{L}} \equiv+1 \mid \sigma^{\prime}(m)\right) \geq \epsilon^{\prime} .
$$

Given $\epsilon>0$, let $T_{L} \geq \frac{\log \epsilon}{\log \left(1-\epsilon^{\prime}\right)}$ be an integer and apply repeatedly the last inequality to time intervals of the form $[m, m+1]$ for integers $m \in\left[0, T_{L}\right)$ : 
$\mathbb{P}\left(\right.$ at some time $\left.t \in\left[0, T_{L}\right],\left.\sigma^{\prime}\right|_{B_{L}} \equiv+1\right) \geq 1-\left(1-\epsilon^{\prime}\right)^{T_{L}}>1-\epsilon$.

Lemma 4.5. If $\rho^{+}>0$, then

$$
\lim _{L \rightarrow \infty} \mathbb{P}\left(B_{l} \text { is captured for some } l \leq L\right)=1 .
$$

Proof. By Proposition 4.1 with $p=\rho^{+}$, pick a sequence of increasing box sizes $L_{i} \in \mathbb{N}$ such that $L_{1}<L_{2}<\ldots$ and

$$
\mathbb{P}\left(B_{L_{i}} \text { is not entrapped }\right)<\frac{1}{i^{2}} .
$$

By the Borel-Cantelli Lemma, almost surely, all but finitely many boxes $B_{L_{i}}$ are entrapped. Now the probability that each box $B_{L_{i}}$ has all corners frozen plus equals

$$
\mathbb{P}\left(B_{L_{i}} \text { has all corners frozen plus }\right)=\left(\rho^{+}\right)^{2^{d}}>0 .
$$

By the Law of Large Numbers, almost surely infinitely many boxes $B_{L_{i}}$ have this property. Combining these statements, almost surely infinitely many boxes $B_{L_{i}}$ are captured, which implies the conclusion of the lemma.

The remaining lemmas will be used in the proof of Theorem 3.2 .

Lemma 4.6. If $\rho^{+}>0$, then

$$
\lim _{M, L \rightarrow \infty} \mathbb{P}\left(B_{L} \text { is } M \text {-captured }\right)=1,
$$

where $M, L$ tend to infinity with no restriction other than $M \leq L$.

Proof. By Proposition 4.1, as in the proof of Lemma 4.5.

$$
\lim _{L \rightarrow \infty} \mathbb{P}\left(B_{L} \text { is entrapped }\right)=1 .
$$

Now for any fixed $L$ and $M \leq L$, let $A_{L, M}$ denote the event that there exist frozen plus spins within distance $M$ from each of the $2^{d}$ corners of $B_{L}$ in every one of the $d$ coordinate directions, as in Definition 4.3 . Thus the event that $B_{L}$ is $M$-captured is the intersection of $A_{L, M}$ with the event that $B_{L}$ is entrapped. The probability of the event that any specific collection of $M$ vertices contains no frozen plus spins is $\left(1-\rho^{+}\right)^{M}$, so

$$
\mathbb{P}\left(A_{L, M}\right) \geq\left(\left[1-\left(1-\rho^{+}\right)^{M}\right]^{d}\right)^{2^{d}}
$$

and this tends to 1 as $M \rightarrow \infty$ (for fixed $d$ ) uniformly in $L \geq M$.

Definition 4.4. Let $B$ be a box of the form $B_{L}(x)$. We say that $B$ is $M$-good if $B$ is $M$-captured and contains no frozen minus vertices. We define $B[M]$, the $M$-trimming of $B$ as

$$
B \backslash\left(\bigcup_{i=1}^{2^{d}} \bar{C}^{i}(M)\right),
$$

where each $\bar{C}^{i}(M)$ is a cube within $B$ containing exactly $M^{d}$ vertices including the $i^{\text {th }}$ corner of $B$ - see Figure 3 for the case $d=2$. 


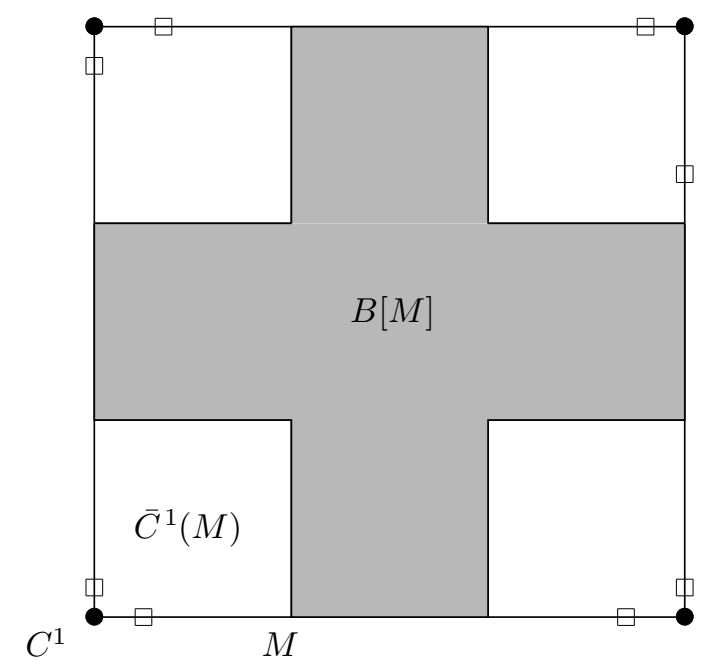

Figure 3: $B[M]$ (grey), the $M$-trimming of an $M$-good box $B$.

Lemma 4.7. Given $\rho^{+}>0$ and $\epsilon>0$, there exist $L<\infty$ and $M<L$ such that for all sufficiently small $\rho^{-}$(depending on $d, L, M, \epsilon$ and $\rho^{+}$),

$$
\mathbb{P}\left(B_{L} \text { is } M \text {-good }\right)>1-\epsilon .
$$

Proof. By Lemma 4.6, we may choose $L, M$ with $M<L$ so that

$$
\mathbb{P}\left(B_{L} \text { is } M \text {-captured }\right)>1-\frac{\epsilon}{2} .
$$

We may also pick $\rho^{-}$small enough so that the probability that $B_{L}$ contains any frozen minus vertices is less than $\frac{\epsilon}{2}$. Thus

$$
\mathbb{P}\left(B_{L} \text { is not } M \text {-good }\right)<\epsilon / 2+\epsilon / 2=\epsilon .
$$

Lemma 4.8. Let $M<L$ and $E_{M, L}$ be the event that $B_{L}$ is $M$-good. Then

$$
\mathbb{P}\left(\text { for any } \sigma^{\prime}(0) \text {, all vertices in } B_{L}[M] \text { fixate to }+1 \mid E_{M, L}\right)=1 \text {. }
$$

Proof. If $B_{L}$ is $M$-good, then by Lemma 4.4 almost surely for some time $t_{0},\left.\sigma^{\prime}\left(t_{0}\right)\right|_{B_{L}} \equiv+1$. In this case, if $M>0,\left.\sigma^{\prime}(t)\right|_{B_{L}}$ need not stay identically +1 for $t>t_{0}$ because vertices near the corners can change from +1 to -1 . But a moment's thought shows that the only vertices near a corner $C^{i}$ that can change to -1 are those in a subset of the cube $\bar{C}^{i}(M)$ - see Definition 4.4. This is because the frozen plus vertices $C^{i, j}$ (from Definition 4.3 protect against the flipping of plus vertices beyond a rectangular parallelipiped contained in $C^{i}(M)$. Note that because $M<L$, every vertex in $B_{L}[M]$ has at least $d+1$ neighbors in $B_{L}[M]$. For example, for $d=2$ (see Figure 3 ), $B_{L}[M]$ is the union of two rectangles each of width at least three (hence at least two) so that every vertex 
in a rectangle has at least three neighbors in the rectangle. Thus $B_{L}[M]$ will have $\left.\sigma^{\prime}(t)\right|_{B_{L}[M]} \equiv+1$ for all $t \geq t_{0}$. Of course the same arguments apply to any translated box $B_{L}(x)=B_{L}+x$ and to $B_{L}[M](x)=B_{L}[M]+x$.

\subsection{Proofs of main results}

The first of the two theorems follows easily from Lemmas 4.4 and 4.5 .

Proof of Theorem 3.1. If the box $B_{L}$ has all $2^{d}$ corners frozen plus, and if at some time $t_{0}$, $\left.\sigma^{\prime}\left(t_{0}\right)\right|_{B_{L}} \equiv+1$, then $\left.\sigma^{\prime}(t)\right|_{B_{L}} \equiv+1$ for all $t \geq t_{0}$. This is because after time $t_{0}$ every vertex in $B_{L}$ (other than the corners whose spin value is frozen) will have at least $d+1$ plus neighbors, so it won't flip sign. If $B_{L}$ is also captured, then by Lemma 4.4 , with probability one, $\left.\sigma^{\prime}(t)\right|_{B_{L}} \equiv+1$ will occur for some $t$. Now by Lemma 4.5, with probability one, $B_{L}$ will be captured for some $L$ and so $\sigma^{\prime}(0)$ will fixate to +1 . The same argument can be translated to $x$ and $B_{L}(x)$ for any $x \in \mathbb{Z}^{d}$.

Proof of Theorem 3.2. We first introduce an auxiliary graph $G$, with vertex set $\mathbb{Z}^{d}$, but where $y_{1}, y_{2}$ are neighbors (with edge $\left.\left\{y_{1}, y_{2}\right\}\right)$ if $\left\|y_{1}-y_{2}\right\|_{\infty}=1$, so every $y$ has $3^{d}-1$ neighbors. Pick $M<L$ to be determined later and tile $\mathbb{Z}^{d}$ with boxes $C_{L}(y)=B_{L}((2 L+1) y), y \in \mathbb{Z}^{d}$. For disjoint such boxes the events of being $M$-good are independent, so the collection of $M$-good boxes and $M$-bad (that is, not $M$-good) boxes defines an independent percolation process on a "renormalized" copy of $G$, called $\hat{G}$, by referring to a vertex $y \in \hat{G}$ as good if $C_{L}(y)$ is $M$-good in $G$ and bad otherwise. Note that when two vertices in $\hat{G}$ are neighbors, this corresponds (for example, for $d=3$ ), to $C_{L}\left(y_{1}\right)$ and $C_{L}\left(y_{2}\right)$ sharing either a face or an edge or just a corner. (The reason for using this notion of connectedness is that a standard (using only nearest neighbor edges) cluster of fixed minus and flipping vertices can extend beyond the standard cluster of $M$-bad (that is, not $M$-good) boxes into the $G$-cluster of $M$-bad boxes and beyond into the $G$-closure of that $G$-cluster.)

Let $\mathcal{C}$ denote the cluster of bad vertices containing the origin in this independent site percolation model of sites in $\hat{G}$, and let $\overline{\mathcal{C}}$ denote its closure (that is, $\mathcal{C}$ unioned with the set of good sites that are $G$-neighbors of sites in $\mathcal{C}$ ). We will show that the $G$-cluster $\mathcal{C}^{*}$ containing the origin, consisting of (eventually) fixed minus vertices together with flipping vertices of $\sigma^{\prime}$, satisfies

$$
\mathbb{P}\left(\mathcal{C}^{*} \subseteq \bigcup_{y \in \overline{\mathcal{C}}} C_{L}(y) \text { for every } \sigma^{\prime}(0)\right)=1
$$

Once Equation (5) has been verified, the proof is completed as follows. By standard percolation arguments, there is some $p^{*}>0$ (one can take, for instance, $p^{*}=1 /\left(3^{d}-1\right.$ ), since $3^{d}-1$ is the number of neighbors of any vertex in $G$ ), such that, if

$$
\mathbb{P}(y \text { is bad })=\mathbb{P}\left(B_{L} \text { is not } M \text {-good }\right)<p^{*},
$$

then there is almost surely no percolation of bad sites and $\mathbb{E}(|\overline{\mathcal{C}}|)<\infty$. To finish the proof we use Lemma 4.7 to choose $\rho^{-}$so small that inequality (6) is valid, and note that by inequality (5),

$$
\left|\mathcal{C}^{*}\right| \leq|\overline{\mathcal{C}}|(2 L+1)^{d}<\infty .
$$


It remains to prove (5), for which we review some old and and introduce some new notation. We refer to vertices $x$ and $y$ in (the original) $\mathbb{Z}^{d}$ as $G$-neighbors if $\|x-y\|_{\infty}=1$ (and we view them as vertices in the graph $G)$. We refer to two boxes $C_{L}\left(y_{1}\right)$ and $C_{L}\left(y_{2}\right)$ as $G$-box-neighbors if $y_{1}$ and $y_{2}$ are $G$-neighbors in (the renormalized) $\mathbb{Z}^{d}$ (and we view these vertices in the graph $\hat{G}$ ). $G$-clusters and $G$-box-clusters (of certain vertices and boxes) and their boundaries will be used later; their definitions are analogous. Note that there exists a $G$-path connecting a vertex in $C_{L}\left(y_{1}\right)$ to one in $C_{L}\left(y_{2}\right)$ and contained within $C_{L}\left(y_{1}\right) \cup C_{L}\left(y_{2}\right)$ iff $C_{L}\left(y_{1}\right)$ and $C_{L}\left(y_{2}\right)$ are $G$-box-neighbors.

A corner region $R(y)$ of a box $C_{L}(y)$ is a cube entirely within $C_{L}(y)$ containing exactly $M^{d}$ vertices including one of the $2^{d}$ corner vertices of $C_{L}(y)$. (A vertex is a corner vertex if it has only $d$ neighbors within $\left.C_{L}(y)\right)$. Any box $C_{L}($.$) contains exactly 2^{d}$ distinct corner regions and their $G$-boundaries are disjoint when $M<L$.

We call a box good if it is $M$-good. If a box is not good, it is bad. We call a vertex bad if it is either in a bad box or in one of the corner regions of a good box; otherwise, it is called a good vertex. Note that by Lemma 4.8 , all good vertices fixate to +1 .

Lemma 4.9. Let $x_{1} \in R_{1}(x)$ and $x_{2} \in R_{2}(x)$ where $R_{1}(x)$ and $R_{2}(x)$ are two distinct corner regions of a good $C_{L}(x)$. Let $\gamma$ be a G-path of bad vertices connecting $x_{1}$ and $x_{2}$; then $\gamma$ contains a vertex $z$ such that $z \notin C_{L}(x)$.

Proof. By way of contradiction, suppose such a $G$-path $\gamma$ exists in $C_{L}(x)$. After translating $C_{L}(x)$ to $\{1,2, \ldots, 2 L+1\}^{d}$, we may assume that $\gamma$ starts at a vertex $\gamma_{1}=\left(a_{1}, a_{2}, \ldots, a_{d}\right)$ such that for all $i, a_{i} \leq M$. If $\gamma$ ends in a different corner region, denote the last vertex in $\gamma$ by $\gamma_{2}=\left(b_{1}, b_{2}, \ldots, b_{d}\right)$ and note that at least for one $i, b_{i}>M+3$. Without loss in generality, assume $b_{1}>M+3$ and note that the $G$-neighbors connecting $\gamma_{1}$ and $\gamma_{2}$ differ in any coordinate by at most 1 . Thus there exists a bad vertex $\gamma_{3}=\left(c_{1}, c_{2}, \ldots c_{d}\right)$ in $\gamma$ with $c_{1}=M+1$. Recall, however, that in a good box only the corner regions contain bad vertices, so that any site $x=\left(x_{1}, x_{2}, \ldots, x_{d}\right)$ with $M+1 \leq x_{i} \leq M+3$ for at least one $i$, is good.

Definition 4.5. For $x \neq y$, two corner regions, $R(x)$ and $R(y)$, of boxes $C_{L}(x)$ and $C_{L}(y)$ are called adjacent if there exists $v \in R(x)$ and $v^{\prime} \in R(y)$ such that $v$ and $v^{\prime}$ are $G$-neighbors.

The proof of the next lemma is straightforward and is left to the reader.

Lemma 4.10. Let $x, y$ be distinct vertices in $\mathbb{Z}^{d}$.

- If there is a $G$-path connecting $R(x)$ and $C_{L}(y)$ and remaining in $C_{L}(x) \cup C_{L}(y)$ then $C_{L}(y)$ has a corner region $R(y)$ adjacent to $R(x)$.

- If $R(x)$ is adjacent to $R(y)$ and $R(y)$ is adjacent to $R(z)$ then $R(x)$ is adjacent to $R(z)$ (transitivity of the adjacency relation).

Lemma 4.11. Let $C_{L}(y)$ be a good box and $C_{L}(x)$ be a bad one. Assume that $C_{L}(y)$ is a $G$-boxneighbor of $C_{L}(x)$. Let $\gamma$ be a $G$-path of bad sites starting in $R(y)$, where $R(y)$ is adjacent to a corner region of $C_{L}(x)$. Then $\gamma$ can only exit $C_{L}(y)$ into either

1. a bad box that is a $G$-box-neighbor of $C_{L}(x)$, or

2. a corner region, adjacent to $R(y)$, of a good box that is a $G$-box-neighbor of $C_{L}(x)$. 
Proof. If the path exits $R(y)$ into a good box $C_{L}(z)$, then it must (by the reasoning of Lemma 4.9 ) enter into a corner region that is adjacent to $R(y)$ and hence by Lemma $4.10, C_{L}(z)$ is a $G$-boxneighbor of both $C_{L}(y)$ and $C_{L}(x)$. If it exits into a bad box, $C_{L}\left(z^{\prime}\right)$, then it must enter into an adjacent corner region $R\left(z^{\prime}\right)$ or into its $G$-boundary, but in either case, $C_{L}\left(z^{\prime}\right)$ is again a $G$-boxneighbor of both $C_{L}(y)$ and $C_{L}(x)$.

The next proposition is an immediate consequence of the previous lemma in the setting where $C_{L}(x)$ is in a $G$-box-cluster of bad boxes.

Proposition 4.12. There is no $G$-path of bad sites from a $G$-box cluster of bad boxes that exits the union of the $G$-box cluster and its $G$-box-boundary.

Proof of Theorem 3.4. The proof uses the version of Proposition 4.1 for Schonmann's modified basic version of bootstrap percolation as discussed in Example 3 of [11. We will focus on the case $d-1=2$. The case $d-1=1$ is easier and can be handled without the use of bootstrap percolation. We leave it for the reader to check that the proof for the cases with $d-1 \geq 3$ proceed essentially the same as for $d-1=2$.

For $d-1=2$, we first partition $\mathbb{Z}^{2} \times\{0,1,2, \ldots K\}$ into disjoint $2 \times 2 \times(K+1)$ pillars $\mathcal{P}_{i, j}=\{(2 i, 2 j),(2 i+1,2 j),(2 i, 2 j+1),(2 i+1,2 j+1)\} \times\{0,1,2, \ldots, K\}$ for $(i, j) \in Z^{2}$. If at any time, all vertices in $\mathcal{P}_{i, j}$ are +1 , then they stay +1 after that since the bottom layer is frozen to +1 , each site in the top layer has 3 neighbors (within the pillar) out of its 5 total neighbors equal to +1 , and all other sites have 4 neighbors (within the pillar) out of its 6 total neighbors equal to +1 .

A pillar $\mathcal{P}_{i, j}$ is fixed +1 in this way at time zero, with probability $\theta^{4 K}$ and the set of such $(i, j)$ are chosen independently of each other. We can now apply the modified Proposition 4.1 as long as we can show that if a pillar $\mathcal{P}_{i, j}$ (at time $t$ ) has at least two neighboring pillars - one in each coordinate direction - all +1 (at time $t$ ), then almost surely by some random time $t+T, P_{i, j}$ will have become all +1 .

This last claim is verified by first arguing, like in the proof of Lemma 4.3 , there is strictly positive probability that $\mathcal{P}_{i, j}$ will be all +1 by the time $t+1$, and then proceeding as in the proof of Lemma 4.4. To explain the first argument, suppose the all +1 neighboring pillars are $\mathcal{P}_{i-1, j}$ and $\mathcal{P}_{i, j-1}$. Then the vertices in the first layer (above the frozen sites), $(2 i, 2 j, 1),(2 i+1,2 j, 1),(2 i, 2 j+$ $1,1),(2 i+1,2 j+1,1)$ can flip to +1 (if they are not already +1 ) in that order followed by the sites in layers $2,3, \ldots, K$ in that order.

Acknowledgments. The authors thank Leo T. Rolla for many fruitful discussions. The research reported in this paper was supported in part by NSF grants DMS-1007524 (S.E. and C.M.N.), DMS-1419230 (M.D.) and OISE-0730136 (S.E., H.K. and C.M.N.). V.S. was supported by ESF-RGLIS network and by Brazilian CNPq grants 308787/2011-0 and 476756/2012-0 and FAPERJ grant E-26/102.878/2012-BBP.

\section{References}

[1] R. Arratia, (1983). Site recurrence for annihilating random walks on $\mathbb{Z}^{d}$. Ann. Probab. 11, 706-713. 
[2] F. Camia, E. De Santis and C. M. Newman. (2001). Clusters and recurrence in the twodimensional zero-temperature stochastic Ising model. Ann. Appl. Probab. 12, 565-580.

[3] M. Damron, H. Kogan, C. M. Newman and V. Sidoravicius. (2013). Fixation for coarsening dynamics in 2D slabs. Electron. J. Prob. 18, 1-20.

[4] M. Damron, H. Kogan, C. M. Newman and V. Sidoravicius, Coarsening dynamics in 2D slabs. To appear Top. Perc. Disord. Sys., 2013.

[5] L. R. Fontes, R. H. Schonmann and V. Sidoravicius. (2002). Stretched exponential fixation in stochastic Ising models at zero-temperature. Commun. Math. Phys. 228, 495-518.

[6] C. D. Howard. (2000). Zero-temperature Ising spin dynamics on the homogeneous tree of degree three. J. Appl. Probab. 37, 736-747.

[7] S. Nanda, C. M. Newman and D. L. Stein. Dynamics of Ising spins systems at zero temperature. In: On Dobrushin's way (From Probability Theory to Statistical Mechanics). R. Milnos, S. Shlosman and Y. Suhov, eds., Am. Math. Soc. Transl. (2) 198, pp. 183-194, 2000.

[8] C. M. Newman, D. L. Stein. Zero-temperature dynamics of Ising spin systems following a deep quench: results and open problems. Physica A. 279, pp. 159-168, 2000.

[9] P. L. Krapivsky, S. Redner and E. Ben-Naim. A Kinetic View of Statistical Physics. Cambridge University Press, 2010.

[10] T. Liggett. Interacting Particle Systems. New York, Berlin: Springer, 1985.

[11] R.H. Schonmann. (1992). On the behavior of some cellular automata related to bootstrap percolation. Ann. Prob. 20, 174-193.

[12] V. Spirin, P. L. Krapivsky and S. Redner. (2001). Freezing in Ising ferromagnets. Phys. Rev. E. 65, 016119-1016119-9. 